\title{
EUV Radiation from B Stars: The Broad Implications for Stellar and Interstellar Astronomy
}

\author{
JOSEPH P. CASSINELLI
}

Astronomy Department, University of Wisconsin, 475 N. Charter St., Madison, WI 53706, USA

Observations made with the Extreme Ultraviolet Explorer (EUVE) of the two bright stars $\epsilon \mathrm{CMa}$ (B2 II) and $\beta$ CMa (B1 II-III) are discussed. The photospheres show excess EUV radiation. The wind of $\epsilon \mathrm{CMa}$ exhibits the Bowen Fluorescence mechanism, along with high ionization stages that help explain the nature of the wind shocks. The pulsation and beat phenomena exhibited by the variable star $\beta$ CMa suggest that deposition of residual pulsation energy might heat and modify the structure of the atmospheres of early-type stars near the $\beta$ Cephei strip. The possibility that many other B stars show a large excess Lyman continuum radiation is considered as a possible source of the ionization of the warm ionized medium (WIM) in the galactic ISM.

\section{Introduction}

A major surprise from $E U V E$ is that the brightest stellar sources in the 500 to 700 $\AA$ range are two $\mathrm{B}$ giant stars, $\epsilon \mathrm{CMa}$ (B2 II) and $\beta \mathrm{CMa}$ (B1 II-III). The stars are bright in the EUV for two reasons. a) The stars lie in a tunnel in the ISM that has a very low HI column density, $\left(N_{H} \approx 1-2 \times 10^{18} \mathrm{~cm}^{-2}\right)$ even though they are at distances of $\approx 200 \mathrm{pc}$. b) The photospheric flux from $\epsilon$ CMa and (to a lesser extent) $\beta \mathrm{CMa}$ exceed the predictions of the model atmospheres that adequately fit visual and UV energy distributions.

The first year of EUVE observations (AO1) of $\epsilon$ CMa are described by Cassinelli et al. (1995, Paper I). Here we present AO2 observations of $\epsilon \mathrm{CMa}$ and results from both $\mathrm{AO} 1$ and $\mathrm{AO} 2$ observations of $\beta \mathrm{CMa}$. The $\beta \mathrm{CMa}$ results will be discussed in an upcoming paper by Cassinelli et al. (1995, Paper II).

Several papers at this meeting are also concerned with the interpretation of the EUVE data from $\epsilon \mathrm{CMa}$ and $\beta \mathrm{CMa}$. The goal is of this review is to provide a broad overview of the significance of the findings to the the fields of stellar atmospheres, pulsation theory, stellar winds and the galactic interstellar medium.

\section{The EUVE Spectra of B Stars from 300 to $730 \AA$}

Figures $1 \mathrm{~A}$ and $1 \mathrm{~B}$ show a comparison of the EUVE LW spectra of the two stars. The results for $\epsilon \mathrm{CMa}$ in Figure $1 \mathrm{~A}$ were surprising for several reasons. The photospheric Lyman continuum radiation is a factor of about 30 higher than predicted from model atmospheres. The photospheric radiation is seen on both sides of the He I ionization edge at $504 \AA$. The star shows strong emission at both the He II Ly $\alpha$ at $304 \AA$ and the resonance line of O III at $374 \AA$. Also, as is discussed in paper I, emission lines from high ion stages from Fe IX to Fe XVI are present in the SW and MW spectra. The presence of these high ionization species provides new information regarding the wind shocks and $\mathrm{X}$-rays from this star. Figure $1 \mathrm{~B}$ shows the LW spectrum of $\beta \mathrm{CMa}$. The spectrum longer than $\lambda 504$ is similar to that of $\epsilon$ CMa, but the count rate is somewhat smaller because the star has a larger column of interstellar attenuation, twice that of $\epsilon \mathrm{CMa}$ 

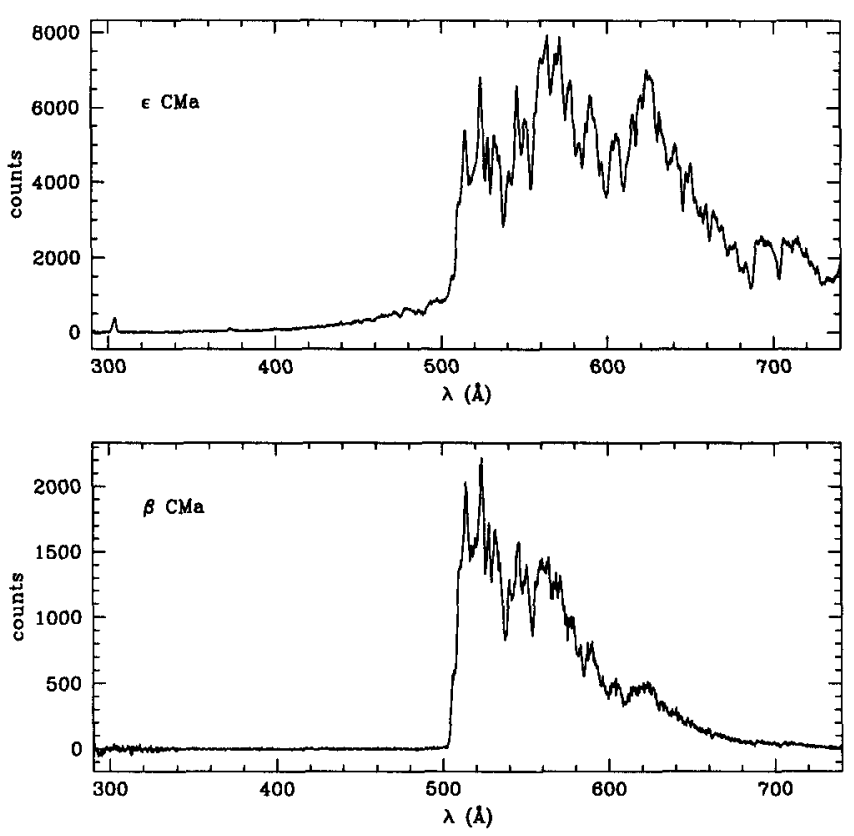

FIGURE 1. Shows $E U V E$ spectra in counts (per $0.27 \AA$ bin) versus wavelength as obtained with the long wavelength (LW) spectrometer on EUVE. (A) The photospheric spectrum of $\epsilon \mathrm{CMa}$ obtained during $\mathrm{AO} 2$ during an observation with an exposure time of 122,244 seconds is shown in the top panel. Stellar wind emission lines of He II at $304 \AA$ and of O III at $374 \AA$ can also be seen. (B) The photosphere of $\beta \mathrm{CMa}$ as observed with an exposure time of 104,575 seconds is shown in the bottom panel.

and the star has a smaller photospheric EUV excess. The star $\beta$ CMa does not show any photospheric radiation shortward of the $\mathrm{He}$ I edge, mostly because of the additional Helium ISM attenuation in its direction. The spectra of this star are especially interesting because of their variability.

Figure $2 \mathrm{~A}$ and $2 \mathrm{~B}$ show portions of the LW spectra for the 2 stars. There are important contribution to the absorption line spectra from both the photospheres and from the stellar winds. The broad absorption troughs are from photospheric line blends. The lines formed in the winds of hot stars tend to be of higher ionization stages because of the $\mathrm{X}$-rays formed in shocks embedded in the winds, and in both spectra, we see absorption by the resonance line of OV at $630 \AA$. Since the resolution of the LW spectrometer of about $1200 \mathrm{~km} / \mathrm{s}$, is larger than the observed terminal speed of the wind, we should not expect to see a wind P-Cygni profile. The spectrum of $\beta \mathrm{CMa}$ is very similar to that from $\epsilon$ CMa but somewhat noisier because of its lower count rate. Both of the spectra shown in Figure 2 were obtained using the dithering procedure to reduce fixed pattern noise. 

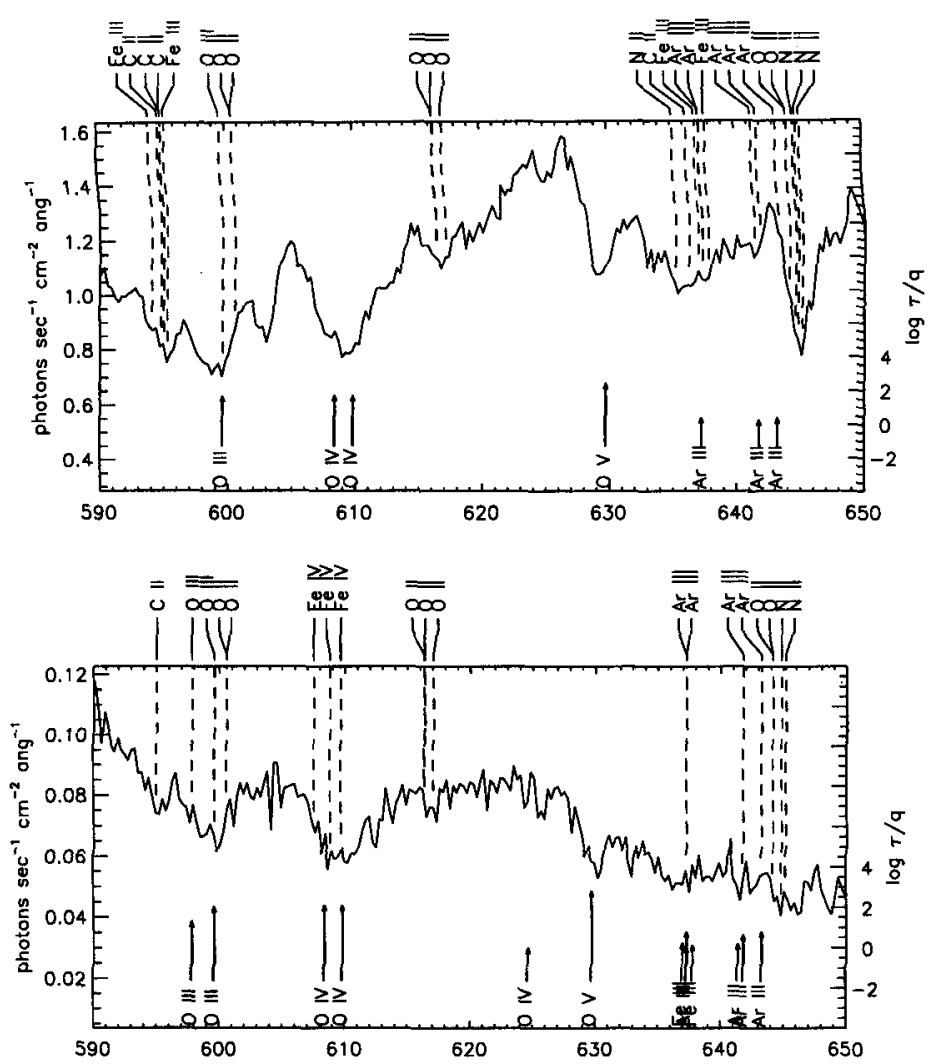

Figure 2. The absorption line spectra of (A) $\epsilon \mathrm{CMa}$ and (B) $\beta \mathrm{CMa}$ in the wavelength band 590 to $650 \AA$. On each figure are two sets of line identifications derived from models. On the top of each figure are shown model photosphere line identifications. On the bottom the arrows show lines that may be prominent in the winds of the stars.

\section{The Photospheric EUV Flux}

Hubeny is presenting a review comparing the observed EUV continua with B star models, so here I will just make comments needed for our ISM and pulsation effects discussions. In the case of $\epsilon \mathrm{CMa}$, the EUV photospheric radiation exceeds the predictions of model atmospheres by more than an order of magnitude. In the case of $\beta \mathrm{CMa}$, the result is less straight forward, but if we accept the the effective temperature derived from a model that fits the UV and visual flux distribution, then this star also has an EUV excess (by a factor of about 5). Since the vast majority of B stars have their effective temperatures determined from fits to observable flux distributions, I am choosing to consider in this review that both $\epsilon \mathrm{CMa}$ and $\beta$ CMa show an excess. The derived effective temperatures of the two stars are $21000 \mathrm{~K}$ for $\epsilon \mathrm{CMa}$, and $23250 \mathrm{~K}$ for $\beta \mathrm{CMa}$.

In paper I, we considered two possible causes of the EUV excess in $\epsilon$ CMa: it is a non-LTE population effect, or it is caused by an enhancement in the temperature in the outermost regions of the photosphere where the EUV radiation forms. We concluded in favor of the higher temperature explanation because the star also shows a infrared 
continuum excess in the near 10-25 $\mu$ m spectral region. Both infrared radiation in that band and the Lyman continuum radiation form at about the same heights in the atmosphere. However, in the IR the opacity is free-free absorption and the source function is the Planck function, and hence the IR flux is sensitive to the temperature, but not to NLTE effects. The temperature must be about $1500 \mathrm{~K}$ higher in the continuum formation region of $\epsilon \mathrm{CMa}$ to explain both the factor of 30 excess in the EUV flux and the roughly $13 \%$ excess at $12 \mu \mathrm{m}$. The reason the temperature has a largely different effect on the emergent fluxes in the two spectral regions is that the EUV is on the Wien side of the spectrum, while the IR is on the Rayleigh Jeans side. So, for $\epsilon$ CMa, the EUV flux varies roughly as $T^{13}$, while the IR flux is approximately proportional to $T$. Because both stars have an IR excess (to a lesser extent in $\beta \mathrm{CMa}$ ) we are currently investigating the possible causes of heating in the outer photospheres of $B$ stars.

\section{Line Emission and Fluorescence in B Star Winds}

Over the past several years a new picture has been developed regarding the structure of the winds of hot stars. Line driven winds are unstable and a sinusoidal variation imposed on the velocity distribution will grow to form shock structures (Owocki et al. 1988). As has recently been shown by Cooper (1994), the shocks generated from the instabilities produce a range of temperatures in the wind and the hottest gas gas gives rise to observable X-ray emission. The heated regions emit most of their energy in the form of spectral lines, many of which occur in the EUV. X-rays from $\epsilon$ CMa are detected by ROSAT, (Drew et al. 1994) and we see in our spectra of the star, lines from Fe IX $(171 \AA)$, Fe XI (181 $\AA)$, Fe XV (284 $\AA)$, Fe XVI (335 $\AA)$. From an analysis of both the $\mathrm{X}$-ray counts and the EUV line radiation, Cohen et al. (1995) conclude the following: the EUV lines confirm that the X-rays are produced thermally in the stellar winds as opposed to non-thermally; The X-ray and especially the EUV photons are subject to wind attenuation; the X-ray energy distribution and the EUV lines from high ions, are consistent with a power law distribution of the emission measure versus temperature; and the power law is of the form expected from the shocked wind theory of Cooper (1994). The simultaneous analysis of X-ray and EUV data provides much more detailed information about shocks and their hard radiation than would be obtainable with either spectral range alone.

MacFarlane et al. (1994) have shown that the X-rays and EUV high ion line emission from shocks have a major effect on the ionization structure in B star winds. In particular they find from a model of $\epsilon \mathrm{CMa}$ that the ionization fraction of $\mathrm{He}^{++}$changes from being a trace ion with abundance of $10^{-4}$ of all Helium, to being a major ion species with a $\approx 0.3$ fractional abundance. With $\mathrm{He}^{++}$being abundant, the origin of the $\lambda 304 \AA$ Ly $\alpha$ line can be explained as from the recombination of $\mathrm{He}^{++}$in the wind. The line is very thick in the wind, and in the multiple scattering transfer of the line radiation it resonantly excites $O$ III. The cascade from the excited level produces O III lines at 3444 $\AA, 3760 \AA$, and the final step leads to the resonance line of O III at $374 \AA$ line, which we see in our spectra of $\epsilon \mathrm{CMa}$. This is of special interest because we are seeing for the first time in a stellar spectrum the dominant transitions of the Bowen Fluorescence mechanism. Bowen (1935) showed that the fluorescence with He II Ly $\alpha$ could explain the anomalously large strengths of O III lines at $3444 \AA$ and $3760 \AA$ seen in planetary nebulae spectra. In MacFarlane's paper at this meeting, he shows that the $\lambda 304$ and $\lambda 374$ lines, are formed by a degradation of the X-ray and EUV high ion radiation in much the same way that $\mathrm{H} \alpha$ is produced by the degradation of stellar Lyman radiation 
in a gaseous nebula. The $E U V E$ observations are thus providing fascinating new insight regarding the nebular aspects of winds.

\section{Ionization of the Galactic ISM with B Star Emission}

Using the estimates of effective temperatures derived from UV and visual continuum studies, we find that both of the B stars bright enough to be detected by the EUVE LW spectrometer show an excess. Although the sample is small, we should ask what would be the effect if all B stars had an average of a factor of 10 excess in their EUV continuum?

A possible application concerns the ionization of the warm ionized medium (or WIM). Reynolds (1991a,b) observes diffuse radiation from the galaxy at both $\mathrm{H} \alpha$ and at $\mathrm{He} \mathrm{I}$, $5876 \AA$. Although $\mathrm{O}$ stars in the galaxy emit sufficient radiation to produce the observed WIM ionization, they suffer from two problems. The WIM radiation comes from gas with a mean height above the galaxy of $1 \mathrm{kpc}$. There is some doubt that $O$ stars could be the source of the ionization of the gas at that height above the plane, basically because the $O$ stars remain close to the material from which they formed so that too little of their radiation would penetrate to the WIM region. Secondly, even if the radiation could do so, it may be too hard to explain the WIM ionization. Tufte \& Reynolds (1995) find that the He II/ H II recombination radiation of the WIM derived from $(\lambda 5876 / \mathrm{H} \alpha)$, is only $1 / 4$ th the ratio observed from HII regions around $O$ stars. According to Reynolds (private communication) if $\mathrm{B}$ stars emitted a factor of 10 more EUV radiation than model atmospheres predict it would go a long way toward solving the WIM problem. The B stars are not so tightly confined to their natal clouds and the ratio of He II / H II produced in B star circumstellar nebulae is more like that seen in the WIM. Having an excess EUV luminosity by a factor of 10 would not affect the effective temperatures of $\mathrm{B}$ stars significantly, because the radiation emitted in the EUV region is a negligible fraction of the total luminosity of a B star.

Since we are not likely to find more B stars with the EUVE that show the strong EUV continua of $\epsilon \mathrm{CMa}$ and $\beta \mathrm{CMa}$, how can we determine if other $\mathrm{B}$ stars emit an excess of EUV radiation? Traditionally the EUV radiation from hot stars is studied by observing the ionization of circumstellar HII regions. Kutyrev \& Reynolds (1995) have compared the radiation from HII regions around B stars with model predictions and have found that two stars (Spica and 139 Tau) out of eight show too large an ionized emission measure. The other six stars could also have an excess if they are in regions that are density bounded, i.e., with too little mass to absorb all the light from the central star. As a alternative approach, we planning to use the spectrometers on the ISO satellite to search for IR excesses in B stars. Recall that the IR continuum and EUV continuum form at about the same heights in the atmosphere, so if there is an IR excess, a larger EUV excess accompanies it.

\section{The $\beta$ Cephei Phenomenon As Seen in the EUV Radiation of $\beta \mathrm{CMa}$}

A long standing puzzle in interiors theory has been the cause of the pulsation seen in stars within the narrow region parallel to the main sequence called the $\beta$ Cephei strip. The pulsation has recently been explained. New opacities calculated by Iglesias et al. (1992), showed a peak at a temperature of about $200000 \mathrm{~K}$. Moskalik et al. (1992) and Kiriakides et al. (1992) applied these opacities to $\beta$ Cephei models and found that the pulsational variability of this class of star could be driven by the classical Kappa 
Mechanism. Interior theorists work mostly with mode analyses that predict the periods and modes of oscillation. In the subject of stellar atmospheres there is also interest in the effects of the propagation of the waves through the atmosphere. For example, the propagation of the waves in the cool Mira variables is responsible for the levitation of the atmospheres that is crucial for the formation of grains, and radiative acceleration of the grains then produces the massive dust driven winds from the stars (Bowen \& Willson 1991).

Our pair of B stars is ideal for studying the atmospheres and envelopes of the $\beta$ Cephei class of variables. The star $\beta$ CMa is the brightest of the $\beta$ Cephei stars and at a temperature of $23250 \mathrm{~K}$, it lies near the red side of the narrow $\beta$ Cephei strip in the HR diagram. The star $\epsilon \mathrm{CMa}$ lies just beyond the redward edge of the strip. The two stars have nearly identical properties in regards to their luminosities, X-ray emission, and wind properties. Yet one pulsates and the other is not reported to be a pulsating variable, (although we find $\epsilon$ CMa to show non-periodic variability in our EUV observations). As $\beta \mathrm{CMa}$ is the brightest of the $\beta$ Cephei stars, it was the first of the class to be well studied, and was the first to show beat phenomena (Meyer 1934). It has 3 periods, at $P=6.00,6.03$, and 5.74 hours, and as seen in the amplitude of the variations the pulsations can show constructive and destructive interference, with a period of 49 days in the case of the 6.0 and 6.03 hour periods. The observations of the pulsations are shown in paper II and in contribution to this meeting by Cohen et al. A portion of the $\beta \mathrm{CMa}$ observations is shown in Figure 3. The bottom section of the figure illustrates the beat phenomenon as it can be seen in the EUV. The periods of pulsation that were derived from observations at visual and UV wavelengths have been detected in the EUV. Future EUVE observations are scheduled to better determine the beat phenomenon seen in Figure 3.

The EUV is an ideal spectral range for studying the $\beta$ Cephei phenomenon. This is because the variation that is seen in the light curve is mostly caused by a variation in the effective temperature of the star, and as we noted earlier, the EUV spectral range is especially sensitive to the radiation temperature since it lies on the Wien side of the spectrum for B stars. Therefore the flux amplitude should be greatly enhanced in the EUV. For the UV studies of $\beta$ CMa by Beeckmans \& Burger (1977), the temperature amplitude of the variation was deduced to be $\Delta \mathrm{T}=180^{\circ} \pm 130^{\circ}$. From our EUV studies, Cohen et al. (these proceedings) have derived $\Delta \mathrm{T}=108^{\circ} \pm 31^{\circ}$. The pulsation is thus much better defined. The EUV results are consistent with the UV results, but mostly because of the large error bars in the UV determination. If higher quality UV or visible data were available, a disagreement between the temperature amplitudes in the UV and the EUV might in fact be expected! This is because the EUV continuum is formed at several density scale heights higher in the atmosphere than is the UV continuum. If the temperature amplitude in the EUV would exactly match that in the UV it would mean that there is no damping of the pulsation between the heights of formation in the atmosphere of the two spectral bands. Using the range of the EUV band alone, Cohen et al. find that there may be a somewhat larger amplitude variation at $\lambda<550 \AA$ than there is at $\lambda>550$. The shorter wavelength region forms deeper in the atmosphere so the difference is in the sense that the pulsational amplitude is smaller at larger heights. On the basis of this, we speculate that there may be some damping of the pulsational wave energy in the outer atmosphere. This could presumably lead to an extra source of heating in the case of $\beta \mathrm{CMa}$. What about for $\epsilon \mathrm{CMa}$ where there is an even clearer need for additional heating? In the standard picture for explaining pulsations, the pulsation is driven in the region of the envelope with enhanced opacity, where positive work is added to the star during one pulsation cycle. Whether the pulsation is observable at the 

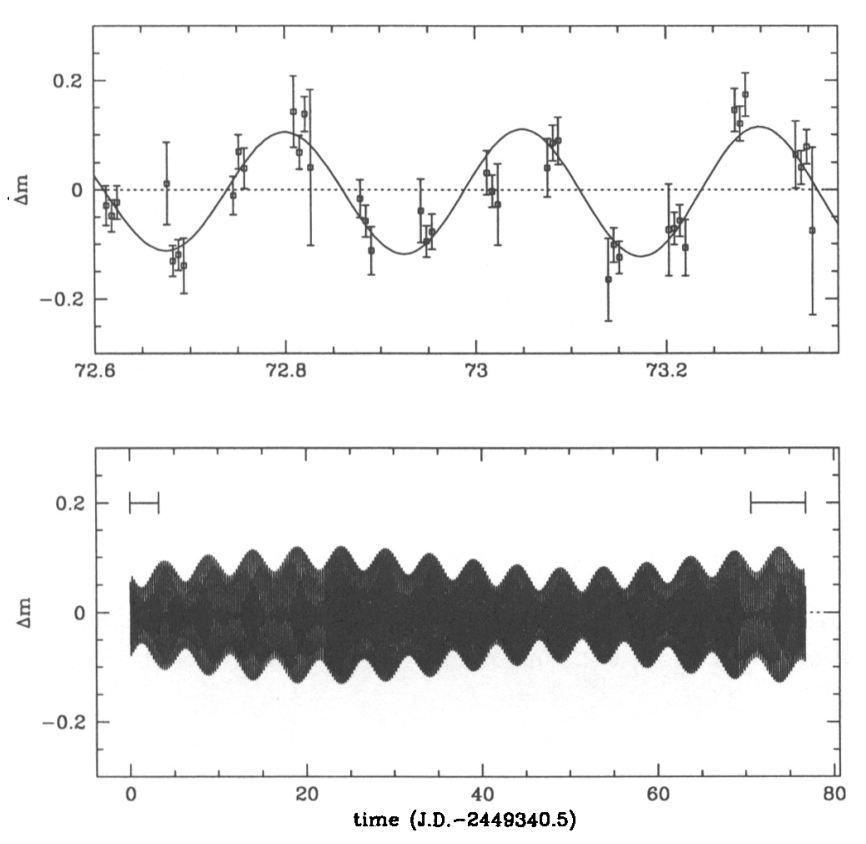

FIgURE 3. The top panel shows the EUV magnitude variation of $\beta \mathrm{CMa}$ versus time during a interval near day 73 in our two part set of observations. In the bottom is shown the beat phenomenon that is derived from the EUVE observations, produced by the three periods of oscillation of this star. The time from one minimum to the next in the interference is about 49 days. The two intervals of our observations are indicated by the two bars at the top of the figure.

top depends to a certain extent on the damping of the pulsation in the $\gamma=5 / 3$ zone above the driving zone. (where $\gamma$ is the ratio of specific heats $c_{P} / c_{V}$ ). Perhaps there is a residual flux of pulsational energy propagating upward also in the case of $\epsilon \mathrm{CMa}$, and it becomes preferentially deposited in the region of formation of the EUV continuum. The possibility that pulsational energy plays a role in the heating of the outer photospheres of these hot stars will be a topic of much further study.

\section{Conclusions}

The goal of this paper has been to highlight the many ways that stellar and interstellar astronomy are affected by the $E U V E$ observations of the anomalously bright B giant stars.

Starting from the interior and progressing outward to the ISM we see the following consequences: In the pulsations of $\beta \mathrm{CMa}$, we now have data for analyzing the possibility that pulsations, driven in a zone in the envelope where $\mathrm{T}=200,000 \mathrm{~K}$, can lead to a change in the structure of the outer atmospheres of stars that lie in or near the $\beta$ Cephei strip. The atmospheres of both $\epsilon \mathrm{CMa}$ and $\beta \mathrm{CMa}$ produce significantly more EUV 
radiation than would be predicted from models that are calibrated by visual and UV energy distributions. From the presence of excess of free-free radiation in the infrared, we have found that the enhanced EUV radiation appears to be due to heating of the outer atmospheres. This diagnostic also suggests that IR observations will be useful to survey other B stars for the presence EUV excesses. The wind of $\epsilon \mathrm{CMa}$ shows a broad range of phenomena not expected before the EUVE launch. There is now a clear link between the production of shocks in the wind with the formation of the EUV lines of high ionization stages. The radiation from these lines and the $\mathrm{X}$-ray emission greatly enhance the abundance of doubly ionized helium, which on recombining leads to the Bowen fluorescence mechanism. This fluorescence had not been seen in a stellar spectrum before. The interstellar medium is also affected by the B star EUV radiation. In the case of $\epsilon$ CMa (or Adhara, as it is referred to in the review by Vallerga and Welsh), the star is responsible for maintaining the ionization of the ISM near the Sun. From a broader perspective the B star EUV radiation may play a role in the ionization of the WIM. The observed $\mathrm{HeI}(\lambda$ 5876) / H $\alpha$ line ratio can be produced by B star radiation, but probably not by that from $O$ stars. Although the sample of $B$ stars that can be observed with $E U V E$ is small, their spectra are leading to an exciting range of investigations.

I thank David Cohen, Joe MacFarlane, Ivan Hubeny and Ron Reynolds for helpful discussions regarding various sections of this review. The research is being supported by NASA grant NAG5-2282.

\section{REFERENCES}

Beeckmans, F., \& Burger, M. 1977, A\&A, 61, 815

BoweN, G. H. \& WILLSON, L. A. 1991, ApJ, 375, L53

BOWEN, I. S. 1935, ApJ, 81, 1

Cassinelli, J. P., Cohen, D. H., MacFarlane, J. J., Drew, J. E., Lynas-Gray, A. E., Hoare, M. G., Vallerga, J. V., Welsh, B. Y., Vedder, P. W., Hubeny, I., \& Lanz, T. 1995, ApJ, 438, 932. (paper I)

Cassinelli, J. P., Cohen, D. H., Drew, J. E., Lynas-Gray, A. E., Hoare, M. G., Hubeny, I., MacFarlane, J. J., Vallerga, J. V., Welsh, B. Y. 1995, ApJ, in preparation (paper II)

Cohen, D. H., Cooper, R. G., Wang, P., MacFarlane, J. J., Owocki, S. P., \& CAssinelli, J. P. 1995, ApJ, in preparation

Cooper, R. G. 1994, Ph.D. Thesis, University of Delaware

MacFarlane, J. J., Cohen, D. H., \& Wang, P. 1994, ApJ, 437, 351

Drew, J. E., DenBy, M., \& HoARe, M. G. 1994, MNRAS, 266, 917

Iglesias, C. A., Rogers, F. J., \& Wilson, B. C. 1992, ApJ, 397, 717

KIRIAKmis, M., EL, ED, \& M. F., Glatzel, W. 1992, MNRAS, 255, 1 P

KutYREV, \& REYNOLDS, 1995, preprint

MeYer, W. F. 1934, Publ. A.S.P, 46, 202

Moskalik, P., \& DziembowsKi, W. A. 1992, A\&A, 256, L5

Owocki, S. P., Castor, J. I., \& RYBICKI, G. B. 1988, ApJ, 235, 914

REYNolds, R. J. 1991a, in The Interstellar Disk- Halo Connection in Galaxies, Dordrecht: Kluwer, 67

REYNOLDS, R. J. 1991b, ApJ, 372, L17

TUFTE, S. \& REYNOLDS, R. J. 1995, in preparation 